\title{
Task-Dependent Activations of Human Auditory Cortex during Pitch Discrimination and Pitch Memory Tasks
}

\author{
Teemu Rinne, ${ }^{1,3}$ Sonja Koistinen, ${ }^{1}$ Oili Salonen, ${ }^{2}$ and Kimmo Alho ${ }^{1,3}$ \\ ${ }^{1}$ Department of Psychology, FI-00014 University of Helsinki, Helsinki, Finland, ${ }^{2}$ Helsinki Medical Imaging Center, FI-00029 Helsinki University Central \\ Hospital, Helsinki, Finland, and ${ }^{3}$ Advanced Magnetic Imaging Centre, FI-02015 Helsinki University of Technology, Espoo, Finland
}

The functional organization of auditory cortex (AC) is still poorly understood. Previous studies suggest segregation of auditory processing streams for spatial and nonspatial information located in the posterior and anterior AC, respectively (Rauschecker and Tian, 2000; Arnott et al., 2004; Lomber and Malhotra, 2008). Furthermore, previous studies have shown that active listening tasks strongly modulate AC activations (Petkov et al., 2004; Fritz et al., 2005; Polley et al., 2006). However, the task dependence of AC activations has not been systematically investigated. In the present study, we applied high-resolution functional magnetic resonance imaging of the AC and adjacent areas to compare activations during pitch discrimination and n-back pitch memory tasks that were varied parametrically in difficulty. We found that anterior AC activations were increased during discrimination but not during memory tasks, while activations in the inferior parietal lobule posterior to the $\mathrm{AC}$ were enhanced during memory tasks but not during discrimination. We also found that wide areas of the anterior AC and anterior insula were strongly deactivated during the pitch memory tasks. While these results are consistent with the proposition that the anterior and posterior AC belong to functionally separate auditory processing streams, our results show that this division is present also between tasks using spatially invariant sounds. Together, our results indicate that activations of human AC are strongly dependent on the characteristics of the behavioral task.

\section{Introduction}

Neurophysiological studies in primates have shown that frequencies of sounds are represented in a tonotopic manner in auditory cortex (AC) (Merzenich et al., 1975; Kaas and Hackett, 2000) and a similar organizational pattern is seen in humans (Woods et al., 2009). However, in behaving animals spectrotemporal receptive fields of primary AC neurons (Fritz et al., 2005) and the topographic organization of primary and secondary AC (Polley et al., 2006) are not fixed but are changing depending on the behavioral task. Thus, AC processes are not simply determined by physical features (e.g., frequency structure) of sounds but are quickly reshaped according to the current behavioral demands. Although the functional organization of AC is still poorly understood, accumulating evidence from studies in humans and other mammals suggests that $\mathrm{AC}$ is composed of functionally differentiated areas and that, analogously to the visual system, AC is organized in separate processing streams (Romanski et al., 1999; Kaas and Hackett, 2000; Rauschecker and Tian, 2000; Arnott et al., 2004; Lomber and Malhotra, 2008).

\footnotetext{
Received June 25, 2009; revised Aug. 24, 2009; accepted Sept. 15, 2009.

This work was supported by the Academy of Finland (Grant \#210587) and Research Funds of the University of Helsinki. We thank Drs. G. Christopher Stecker and David L. Woods for insightful comments and Ms. Suvi Talja for her skilled assistance in programming data analysis tools. T.R. is the Principal Investigator and carried the main responsibility in designing the experiment, analyzing the fMRI data, and writing the article; S.K. participated in designing the experiment, implemented and performed the experiment, analyzed the behavioral data, and participated in writing; 0.S. participated in the analysis of anatomical MRI data and writing; K.A. participated in designing the experiment and writing the article.

Correspondence should be addressed to Teemu Rinne, Department of Psychology, P0 Box 9, Fl-00014 University of Helsinki, Finland. E-mail: teemu.rinne@helsinki.fi.

D01:10.1523/JNEUROSCI.3012-09.2009

Copyright $\odot 2009$ Society for Neuroscience $\quad$ 0270-6474/09/2913338-06\$15.00/0
}

Several previous functional magnetic resonance imaging (fMRI) studies have shown that activations of human AC are strongly modulated by attention-engaging auditory tasks (Woodruff et al., 1996; Jäncke et al., 1999; Janata et al., 2002; Rinne et al., 2005; Degerman et al., 2006; Rinne et al., 2008; Mayer et al., 2009). The most pronounced attention-related modulations are typically seen in nonprimary parts of AC in the lateral superior temporal gyrus (STG) (Hall et al., 2000; Petkov et al., 2004; Woods et al., 2009). The functional significance of attention-related modulations observed in fMRI is not well understood but it is has been suggested that the increased STG activations during auditory tasks could be related to some additional resources, such as working memory (Petkov et al., 2004; Brechmann et al., 2007), required by active listening tasks. Previous studies have also reported differences in the distribution of human AC activations during spatial and nonspatial auditory tasks. Typically these studies show stronger activations associated with spatial tasks in the posterior STG and inferior parietal lobule (IPL) as compared with nonspatial tasks (Alain et al., 2001; Arnott et al., 2004; Barrett and Hall, 2006). The distribution of activations during nonspatial tasks, however, seems less consistent in the literature. While anterior AC appears to be involved in tasks requiring pitch or pitch pattern discrimination, some studies suggest that also areas in the posterior STG are activated in such auditory nonspatial tasks (Patterson et al., 2002; Warren and Griffiths, 2003; Arnott et al., 2004; Barrett and Hall, 2006).

Previous results clearly demonstrate that processing of sounds in $\mathrm{AC}$ depends on the characteristics of a behavioral task. However, the task dependence of AC activations has not been systematically studied. In the present study, we applied high-resolution 

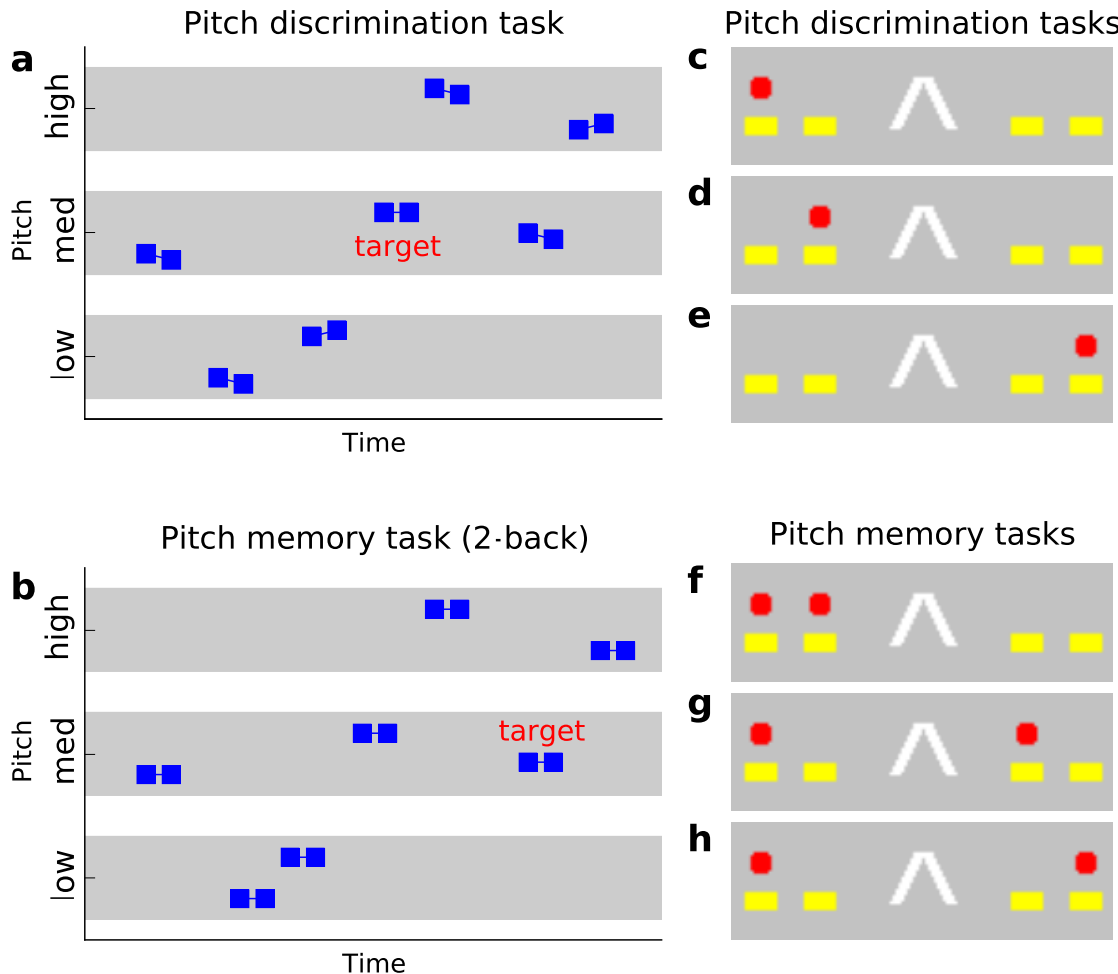

Figure 1. $\quad \boldsymbol{a}, \boldsymbol{b}$, During all auditory and visual task blocks, low (lowest frequency $200 \mathrm{~Hz})$, medium $(561 \mathrm{~Hz})$, and high $(1573 \mathrm{~Hz})$ pitched tones were presented with six equidistant pitch levels in each category (pitch range within a category depended on subjective pitch discrimination performance during training). The tones were $200 \mathrm{~ms}$ in duration and consisted of two successive $100 \mathrm{~ms}$ parts (each part included a $5 \mathrm{~ms}$ linear onset and offset ramps). In pitch discrimination tasks (a), the last half of each tone was slightly lower or higher in pitch than the first part (the magnitude of pitch difference depended on the difficulty level). Subjects were required to press a button when the two halves of a tone had the same pitch (target). In the pitch memory tasks $(\boldsymbol{b})$, the two halves of a tone always had the same pitch and subjects were required to respond when a tone belonged to the same pitch category as the one presented one, two, or three trials before (target in a 2-back task is illustrated). $\boldsymbol{c}-\boldsymbol{h}$, The task and its difficulty level were indicated by task instruction symbols presented on a screen from 6 s before each block onset until the end of the block. A letter " $\Lambda^{\text {" }}$ (Lambda) or " $V$ " (not shown in the figure) in the middle of task instruction symbols indicated the task modality, auditory or visual, respectively. Pitch discrimination tasks were indicated by one red dot $(\boldsymbol{c}-\boldsymbol{e})$, while the pitch memory tasks were indicated by two red dots $(\boldsymbol{f}-\boldsymbol{h})$. In the pitch discrimination tasks, task difficulty level was indicated by the position (yellow rectangles) of the red dot (the leftmost position, easy; second position from the left, medium; rightmost position, hard). For the pitch memory tasks, the distance between two red dots indicated the relative serial positions of the sounds to be compared. For the visual tasks, in addition to the letter $V$, two red dots were presented at second and third position from the left.

imaging of the $\mathrm{AC}$ and adjacent areas to compare activations during pitch discrimination and n-back pitch memory tasks that required auditory attention and were varied parametrically in difficulty (see Fig. 1). We also measured activations during a visual task with sounds similar to those used in the auditory tasks.

\section{Materials and Methods}

Subjects. Subjects $(N=17,13$ women, all right-handed) were 23-30 years (mean 25 years) of age. All subjects had normal hearing, normal or corrected-to-normal vision, and no history of psychiatric or neurological illnesses. An informed written consent was obtained from each subject before the experiment. The study protocol was approved by the Ethical Committee of the Hospital District of Helsinki and Uusimaa, Finland.

Stimuli and tasks. Subjects were presented with tones (diotic presentation, duration $200 \mathrm{~ms}$, onset-to-onset interval $0.8-1 \mathrm{~s}$ ) consisting of two successive $100 \mathrm{~ms}$ parts (each part included a $5 \mathrm{~ms}$ linear onset and offset ramps). The tones formed low (lowest frequency $200 \mathrm{~Hz}$ ), medium $(561 \mathrm{~Hz})$, and high $(1573 \mathrm{~Hz})$ pitch categories each including six equidistant pitch levels. During pre-fMRI training, the pitch range within each category was adjusted individually for each subject depending on their pitch discrimination ability (highest possible frequencies were 320, 898 and $2517 \mathrm{~Hz}$ in low, medium, and high category, respec- tively). Thus, pitch ranges within a category varied depending on subjective performance but each category always contained six equidistant pitch levels. In pitch discrimination tasks (Fig. 1a), the second half of each tone was one, two, or three levels (depending on the difficulty level) lower or higher in pitch than the first half. Subjects were required to attend to the tones and indicate by pressing a button with their right hand when the two halves of a tone had the same pitch. In pitch memory tasks (Fig. $1 b)$, the tones were otherwise similar, but the two halves of a tone always had the same pitch, and the subjects indicated with a button press when a tone belonged to the same pitch category as the one presented one, two, or three trials before.

The tones were delivered with an UNIDES ADU2a audio system (Unides Design) via plastic tubes through a porous EAR-tip (ER3, Etymotic Research) acting as an earphone. The noise of the scanner was attenuated by the earplugs, circumaural ear protectors, and viscoelastic mattress inside and around the headcoil and under the subject. The experiment was controlled using Presentation software (Neurobehavioral Systems).

The sounds were presented in $15 \mathrm{~s}$ blocks alternating with $8 \mathrm{~s}$ breaks with no sound stimuli. During the breaks, the subjects focused on a fixation mark (white $\mathrm{X}$ on gray background, red 186, green 186, blue 186) presented for $2 \mathrm{~s}$ in the middle of a screen (viewed through a mirror fixed to the head coil). The fixation mark was replaced by nonverbal taskinstruction symbols $6 \mathrm{~s}$ before the start of the next block (Fig. $1 c-h$ ). The task-instruction symbols were presented until the end of the block. For each task type and difficulty level (two auditory tasks with three difficulty levels and one visual task), 15 blocks were presented resulting in 105 blocks altogether. There were 2-4 targets in each block.

In the visual task, the subjects were instructed to ignore the sounds and to detect occasional slight luminance changes of a flickering gray rectangle (R 186, G 186, B 186) underlying the task-instruction symbols (Fig. $1 c-h$ ). The target rectangle was slightly brighter (R 194, G 194, B 194). The visual stimulus sequences delivered concurrently with auditory sequences were similar to auditory sequences (stimulus duration $200 \mathrm{~ms}$, onset-to-onset interval $0.8-1 \mathrm{~s}$, 2-4 targets in each block). The auditory sequences presented during the visual conditions were identical to the auditory sequences during pitch memory tasks (i.e., the two halves of each tone had the same pitch).

Pre-fMRI training. To reveal differences in brain activity between easy and hard tasks and between pitch discrimination and pitch memory tasks, the hardest difficulty levels were made intentionally highly demanding. Before the fMRI session, subjects were carefully trained to perform the tasks (1-2 $\mathrm{h}$ of training in two sessions $1-5 \mathrm{~d}$ before scanning). During training, it was emphasized to the subjects that maximum effort in performance is essential especially during the most difficult levels.

Analysis of the behavioral data. Mean hit rates and reaction times were calculated separately for each task. Correct responses occurring between 200 and $1300 \mathrm{~ms}$ from target onset were accepted as hits. Hit rate was defined as the number of hits divided by the number of targets. Mean reaction time was calculated only for hits. Behavioral results were analyzed using one-way repeated-measures ANOVAs with factor Task Difficulty (3 levels). Due to technical problems in data collection, the behavioral data of one subject were lost. Based on good behavioral results 


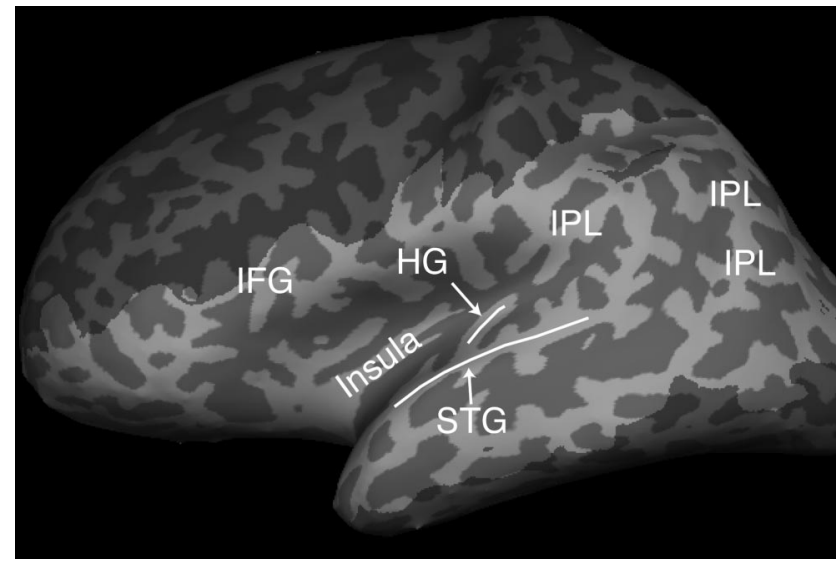

Figure 2. Inflated left-hemisphere cortical surface (light gray, gyri; dark gray, sulci). The areas covered in the present fMRI study are shown in lighter grayscale. The EPI slices were aligned along Sylvian fissures to cover the STG, HG, anterior insula, and most of the IPL of both hemispheres. Due to anatomical differences between left and right hemispheres and between subjects the imaged area did not completely cover the IPL in all cases. This problem was even larger for the inferior frontal gyrus $(\mathbb{F G})$, which was therefore not considered in the present study.

during training, self-reported performance after fMRI and typical brain activation patterns, this subject's fMRI data were not excluded, however.

fMRI data acquisition and analysis. fMRI data were acquired with a 3.0 T GE Signa system retrofitted with an Advanced NMR operating console and a quadrature birdcage coil. Functional images were acquired using a $\mathrm{T} 2{ }^{*}$-weighted gradient-echo echo-planar (GE-EPI) sequence [repetition time (TR), $2000 \mathrm{~ms}$; echo time (TE), $32 \mathrm{~ms}$; flip angle, $90^{\circ}$; voxel matrix, $96 \times 96$; field of view (FOV), $20 \mathrm{~cm}$; slice thickness, $2.1 \mathrm{~mm}$ with no gap; in-plane resolution, $2.1 \mathrm{~mm} \times 2.1 \mathrm{~mm}$; number of slices, 24]. Based on an anatomical scout image (sagittal slices, slice thickness $3 \mathrm{~mm}$, in-plane resolution $0.94 \mathrm{~mm} \times 0.94 \mathrm{~mm})$, the middle EPI slices were aligned along Sylvian fissures (Fig. 2). The functional scanning was divided in two 23 min runs resulting in $\sim 2 \times 690$ images. After the first run, there was a short break during which subjects remained in the scanner and were instructed not to move their heads or speak. After the functional scans, a fluid-attenuated inversion recovery image using the same imaging slices but with denser in-plane resolution was acquired (FLAIR; TR, 10,000 ms; TE, 120 ms; voxel matrix $320 \times 192$; FOV, 20; slice thickness, $2.1 \mathrm{~mm}$; in-plane resolution $0.39 \times 0.39$ ). Finally, at the end of the session, high-resolution anatomical images were acquired (voxel matrix, $156 \times 256 \times 256$; resolution, $1 \times 0.98 \times 0.98 \mathrm{~mm}$ ).

Global voxel-vise analysis was performed using the tools developed by the Analysis Group at the Oxford Centre for Functional MRI of the Brain (FMRIB) and implemented within FMRIB's software library (FSL, release 4.1,www.fmrib.ox.ac.uk/fsl, Smith et al., 2004). First, data from the two runs were combined into one file for motion correction. The motion-corrected data were again split into two separate files, high-pass filtered (cutoff $100 \mathrm{~s}$ ), and spatially smoothed (Gaussian kernel of $7 \mathrm{~mm}$ full-width half-maximum). First-level statistical analysis was performed using FMRIB's improved linear model. Based on the timing information recorded during the experiment, each functional image was labeled as either pitch discrimination (3 difficulty levels), pitch memory (3 levels), visual task, or baseline ( $8 \mathrm{~s}$ breaks with no sound stimuli). The hemodynamic response function was modeled with a gamma function (mean lag $6 \mathrm{~s}, \mathrm{SD} 3 \mathrm{~s}$ ) and its temporal derivative. Contrasts were specified to create Z-statistic images testing for task and difficulty effects. A second-level statistical analysis using fixed-effects combined the data from the two runs.

For analysis across participants (third level analysis), the data were anatomically normalized in the following steps: First, cortical surfaces were extracted from high-resolution anatomical images, transformed to spherical standard space, and anatomically normalized on the basis of the cortical gyral and sulcal patterns using FreeSurfer (v4.0.5, http://surfer. nmr.mgh.harvard.edu). Next, the three-dimensional (3D) spherical cortical surfaces were rotated and projected to a two-dimensional (2D) space separately for each hemisphere using equal area Mollweide projection (Python libraries matplotlib and basemap, http://matplotlib.sourceforge.net). This procedure produced 3D-to-2D anatomical transformation matrices for each subject that were then applied separately for each subject to transform the results of the 3D second-level statistical analysis to 2D.

Finally, the group analysis (FMRIB's local analysis of mixed effects using automatic outlier deweighting, $N=17$ ) was run on these flattened data. $Z$-statistic images were thresholded using clusters determined by $Z$ $>2.3$ and a (corrected) cluster significance threshold of $p<0.05$ (using Gaussian random field theory).

\section{Results \\ Behavior}

Hit rate (HR) across all auditory tasks was 64\% and mean reaction time (RT) $705 \mathrm{~ms}$ ( $N=16$, see Materials and Methods). HRs decreased with increasing task difficulty both in pitch discrimination tasks $\left(F_{(2,30)}=21, p<0.0001\right.$, linear trend, $F_{(1,15)}=44$, $p<0.0001)$ and pitch memory task $\left(F_{(2,30)}=62, p<0.0001\right.$, linear trend, $\left.F_{(1,15)}=365, p<0.0001\right)$, where increasing difficulty also decreased RTs $\left(F_{(2,30)}=3.3, p=0.050\right)$, linear trend, $\left.F_{(1,15)}=5.6, p=0.032\right)$.

\section{Voxel-vise analysis of $\mathrm{fMRI}$ data}

AC activations to sounds in the absence of auditory attention were isolated by contrasting activations during the visual task with activations during the $8 \mathrm{~s}$ breaks with no sounds. In both hemispheres, widespread AC regions, including the anterior and posterior STG and anterior insula, were activated by unattended sounds (Fig. 3a, blue and yellow; for anatomical labels, see Fig. 3g; for results in individual subjects, see supplemental Fig. 1, available at www.jneurosci.org as supplemental material). General effects of auditory tasks were isolated by contrasting all auditory tasks with the visual task. Distinct activation clusters were detected in the posterior STG, IPL, and insula in both hemispheres and in the left anterior STG (Fig. $3 a$, red and yellow).

Activations specific to the two auditory tasks were extracted by comparing each auditory task (all difficulty levels) with activations during the visual task. Enhanced activations related to pitch discrimination were detected bilaterally in the anterior STG, posterior STG, and anterior insula (Fig. 3b, blue and yellow). Activation increases during pitch memory tasks, in turn, were found bilaterally in the posterior STG, IPL, and anterior insula (Fig. 3b, red and yellow). Direct comparison of the two types of auditory tasks with each other revealed stronger activations in wide temporal and insular areas during pitch discrimination than during pitch memory tasks (Fig. $3 c$, blue), while areas in the IPL and insula were more activated during pitch memory than during pitch discrimination tasks (Fig. $3 c$, red; for results in individual subjects, see supplemental Fig. 2, available at www.jneurosci.org as supplemental material).

Effects of auditory task-difficulty were examined with linear contrasts. Significant activation increases with increasing task difficulty were detected in the anterior insula and IPL for pitch memory (Fig. 3d, red and yellow), but significant activations (not shown) were detected only in the right insula for pitch discrimination. However, with a more lenient threshold $(Z>1.64$, corresponding to uncorrected $p=0.05$ ) distinct clusters showing higher activity for more difficult discrimination tasks (Fig. 3d, yellow) were seen within the insula and IPL, in areas where activations were also modulated by memory load. (Note that in Fig. $3 d$ different thresholds are used for pitch discrimination and pitch memory tasks.)

Separate contrasts were conducted to detect areas where activations were higher during the visual task than during the auditory 


\section{Left hemisphere Right hemisphere}

a

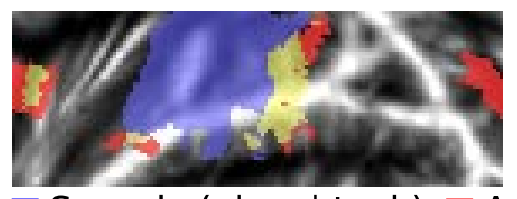

Sounds (visual task)

b
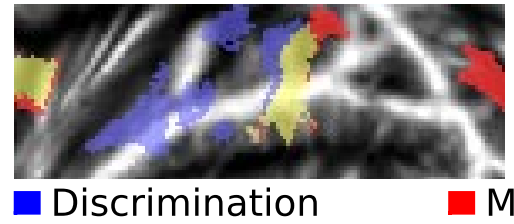

C

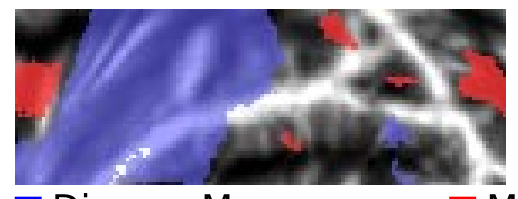

Discr > Memory

d

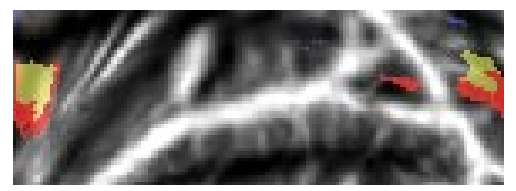

- Discr linear (n.s.)

e

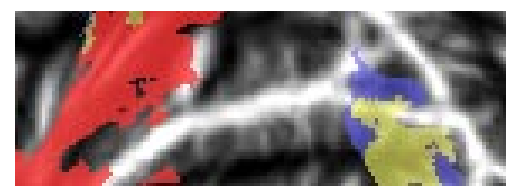

Vis $>$ Discr

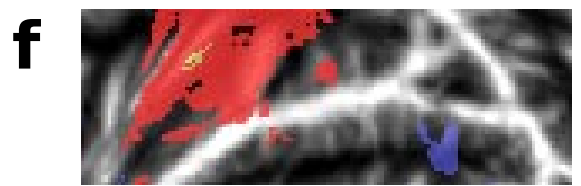

Vis > Memory

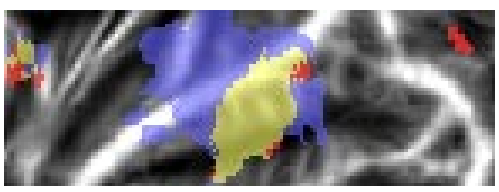

Auditory tasks

Both

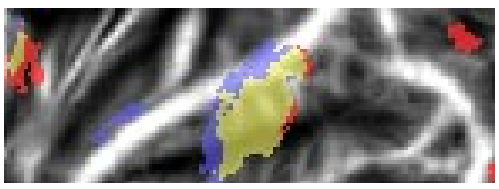

Memory

Both

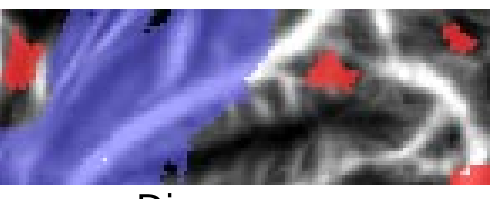

Memory > Discr

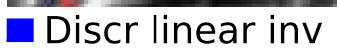

Memory linear inv

g

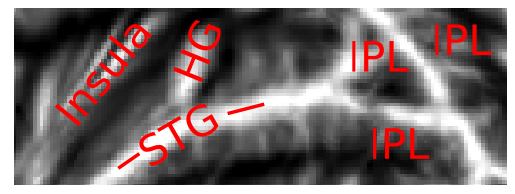

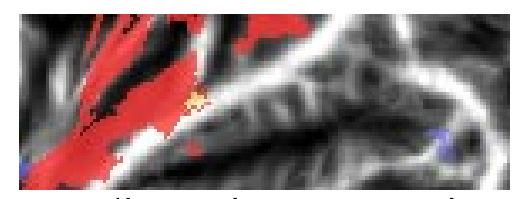

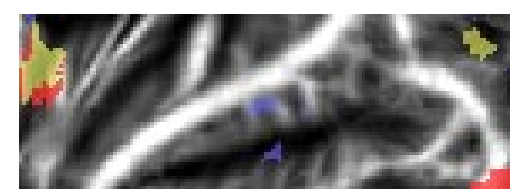

Memory linear
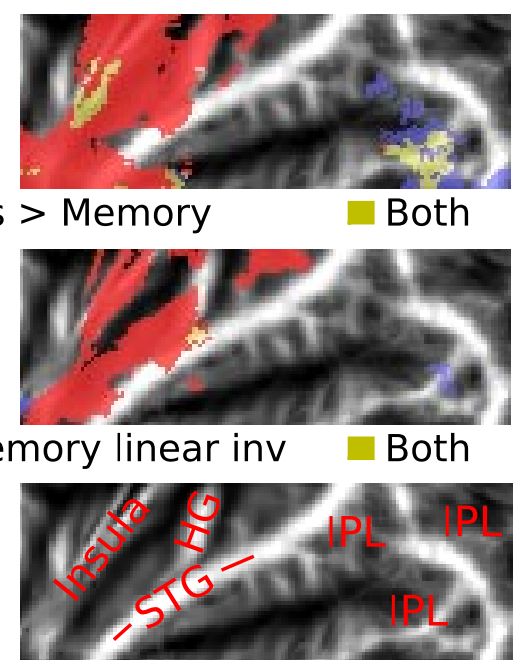

Figure 3. Activations $(\boldsymbol{a}, \boldsymbol{f})$ shown on flattened mean $2 D$ cortical surface $(N=17$, threshold $Z>2.3$, cluster-corrected $p<$ 0.05 unless otherwise specified). $\boldsymbol{a}$, Activations to sounds in the absence of auditory attention (blue) were isolated by contrasting activations during the visual task with activations during the $8 \mathrm{~s}$ breaks with no sounds. General effects of auditory tasks were isolated by contrasting all auditory tasks with the visual task (red). Areas showing significant activations in both contrasts are shown in yellow. $\boldsymbol{b}$, Activations specific to pitch discrimination (blue) and pitch memory (red) tasks were extracted by comparing each auditory task (all difficulty levels) with activations during the visual task. c, Areas where activations were stronger during pitch discrimination than pitch memory tasks (blue) and areas where activations were stronger during pitch memory than pitch discrimination tasks (red). $\boldsymbol{d}$, Effects of task difficulty (linear contrasts) on pitch discrimination (blue, threshold $Z>1.64$ corresponding to uncorrected $p=$ 0.05 ) and pitch memory (red, $Z>2.3$, cluster-corrected $p<0.05$ ) tasks. $e$, Areas where activations were higher during the visual task than during pitch discrimination task (blue) and during pitch memory tasks (red). $\boldsymbol{f}$, Results of linear inverse contrast revealing areas where activations decreased with increasing task difficulty during pitch discrimination (blue) and pitch memory (red). $\boldsymbol{g}$, Anatomical labels: STG (superior temporal gyrus), HG (Heschl's gyrus), IPL (inferior parietal lobule, consisting of angular gyrus and supramarginal gyrus).

tasks. As compared with pitch discrimination, activations were higher during the visual task bilaterally in the IPL and in parts of the insula (Fig. 3e, blue and yellow). As compared with pitch memory tasks, activations were higher during the visual task also in the IPL and, in addition, in wide areas of the anterior temporal lobe and insula bilaterally (Fig. $3 e$, red and yellow). Apparently, these differences were not due to activation increases during the visual task but to activation decreases during the auditory tasks. This was revealed by an inverse linear contrast indicating that activations decreased with increasing memory load (Fig. 3f, red and yellow) in the anterior insula, anterior STG, Heschl's gyrus (HG), and posterior STG. Correspondingly, activations decreased in the IPL with increasing pitch discrimination difficulty (Fig. $3 f$, blue).

\section{Discussion}

As expected, task-irrelevant sounds during the visual task activated areas in the supratemporal cortex including HG (where the primary AC is located) and STG bilaterally (Fig. $3 a$ ). As compared with the visual task, the auditory tasks in general were associated with enhanced activations especially in the posterior/lateral STG (Fig. $3 a$ ). However, activations in the AC and adjacent areas were strongly dependent on whether subjects performed pitch discrimination or pitch memory tasks. We found that activations in the anterior STG increased during pitch discrimination but not during pitch memory tasks while activations in the IPL were increased during pitch memory tasks but not during pitch discrimination, and areas in the posterior STG showed enhanced activations during both kinds of tasks (Fig. 3b).

There is abundant evidence supporting the role of lateral HG and anterior STG in pitch processing (Patterson et al., 2002; Warren and Griffiths, 2003; Penagos et al., 2004; Barrett and Hall, 2006; Krumbholz et al., 2007). Consistently, the present results implicate these areas in active pitch discrimination (Fig. $3 b, c$ ). However, we also found activations associated with pitch discrimination posterior to HG, in the STG including its superior plane, the so-called planum temporale (PT). These activations overlapped partly with activations during pitch memory tasks. The activations shared by pitch discrimination and pitch memory tasks may be related to attentive processing of sounds in general (Jäncke et al., 1999; Petkov et al., 2004; Rinne et al., 2005). However, as both tasks required processing of pitch information, overlapping activations in the posterior STG may also be related to some aspect of active pitch processing. Thus, consistent with the model proposed by Griffiths and Warren (2002), STG areas posterior to HG including the PT might act as a "computational hub" engaged in analysis and segregation of sound patterns and matching incoming and previously stored patterns. Our results are also in line with the suggestion that between the AC areas, there is a 
hierarchy where processing of pitch and pitch variation (including melody) continues, after initial processing in $\mathrm{HG}$, at higher stages in the anterior and posterior STG (Patterson et al., 2002; Hall and Plack, 2009; McLachlan, 2009). The present results show that activations within this hierarchy are strongly dependent on the behavioral task.

We detected activations related to pitch discrimination also in the anterior insula bilaterally (Fig. 3b) (Wong et al., 2004; Gaab et al., 2006). These activations were more distinct during the pitch memory tasks than during the pitch discrimination tasks (Fig. $3 c$ ). However, during both kinds of tasks, these activations increased with increasing task demands (Fig. $3 d$ ). The anterior insula was also activated by the sounds when attention was directed to the visual task (Fig. 3a). Together, these results suggest that the anterior insula has a complex role in auditory processing and that its function is probably not directly related to pitch processing but to some more general aspects of active listening (Bamiou et al., 2003; Lawrence et al., 2003; Deary et al., 2004; Mutschler et al., 2009).

Based on a comparison of activations during auditory 2-back and 0-back memory tasks, it has been suggested that activations in the posterior STG are associated with auditory working memory (Brechmann et al., 2007). In the present study, we found small activation clusters in the posterior STG that showed higher activity during the pitch memory tasks (Fig. $3 b$ ). However, we found no STG areas that were significantly modulated by the memory load (Fig. 3d). Therefore, the posterior STG activations during the present pitch memory tasks were presumably related to attentive processing of sounds in general and not to auditory working memory per se. Previous studies have also implicated IPL in auditory working memory (Martinkauppi et al., 2000; Gaab et al., 2006). One study applied spatial n-back tasks and reported activation increases with increasing memory load in various parietal lobe locations including IPL (Martinkauppi et al., 2000). Consistently, in the present study, we found bilateral IPL activation clusters that were strongly associated with the pitch memory tasks. These IPL clusters showed higher activity during the pitch memory tasks, but not during the pitch discrimination tasks as compared with the visual task (Fig. $3 b$ ), greater activations during the pitch memory tasks than during the pitch discrimination tasks (Fig. 3c), and increasing activity with an increasing memory load (Fig. 3d).

Unexpectedly, we found that wide areas of the anterior AC and anterior insula were strongly deactivated during the pitch memory tasks. In these areas, activations were lower during the memory tasks as compared with the visual task (Fig. 3e) and decreased with an increasing memory load (Fig. 3f). Interestingly, the areas where activations decreased with memory load included HG and areas in the posterior STG. To our knowledge, such decreases in the primary and nonprimary human AC activations associated with an auditory task have not been reported before. These results might be explained by the characteristics of the present auditory tasks applying fast presentation rates (one sound in $0.6-0.8 \mathrm{~s}$ ). In a previous study applying auditory (spatial) n-back tasks with a lower rate (one sound in $3.1 \mathrm{~s}$ ), RTs were significantly longer in a 3-back condition than in 1-back condition (Martinkauppi et al., 2000). In the present study, responses became faster with increasing memory load and the shortest RTs were observed in the 3-back memory task. In addition to an unusually fast presentation rate, the present n-back tasks were complicated by the requirement to categorize sounds as "low," "medium," or "high." Perhaps in the difficult 2-back and 3-back tasks, the subjects had to give their response as quickly as possible to return to rehearsing the categorized sequence of past sounds before perceiving and categorizing the next sound. Although both the pitch discrimination and pitch memory tasks required pitch processing, the present discrimination tasks required detailed pitch analysis of both the first and the last $100 \mathrm{~ms}$ halves of each tone, while the memory task required the categorization of tones into low, medium, or high pitch groups (Fig. 1). During these memory tasks, detailed pitch analysis may have been halted actively; i.e., brain processes involved in pitch analysis may have been deactivated as soon as the pitch category was resolved to save resources and time for the actual memory task. In literature, taskinduced deactivation is commonly defined as relative decrease of activations during active tasks as compared with a "resting" baseline. One theory explaining deactivations observed in such comparisons postulates that these deactivations are caused by interruption of processing that occurs "as default" during the resting state (McKiernan et al., 2006). In line with this theory, we suggest that the present task-dependent deactivations observed during active auditory memory tasks are due to interruption of pitch processing that occurs as default in the AC for each incoming sound. However, these accounts of the present unexpected findings are unavoidably post hoc in their nature and should be carefully tested in future fMRI experiments.

Our results are in line with the suggestion that anterior and posterior AC belong to functionally separate auditory processing streams. We found that activations of AC areas anterior to HG were enhanced during pitch discrimination and decreased during pitch memory tasks. Furthermore, areas showing increased activations during pitch discrimination and memory tasks seemed at least partially segregated: while HG and areas of the anterior STG showed enhanced activations only during pitch discrimination, activity in the IPL was more strongly associated with the pitch memory task. However, unlike in previous studies suggesting spatial and nonspatial processing in the posterior and anterior processing streams, respectively, the present anterior-posterior differences in AC activations were observed between pitch discrimination and pitch memory tasks both requiring analysis of pitch information in spatially stationary sounds.

As discussed above, in the present and previous studies, IPL activations were modulated by memory load (Martinkauppi et al., 2000). Interestingly, in the present study, IPL activations seemed to increase also with increasing demands in the pitch discrimination task (Fig. $3 d$ ), although this effect was seen only with a more lenient statistical threshold. Together, these results suggest that the present IPL activations were related to auditory task difficulty.

In conclusion, while the present results are partly consistent with the prevailing dual-stream model of auditory processing assuming an anterior "what" and posterior "where" pathway, our results suggest that this division is not necessarily only between spatial and nonspatial processing but that similar anterior-posterior division is present also between auditory tasks with spatially invariant sounds. The present results suggest that areas in the posterior AC are involved in tasks that cannot be completed within the default-mode processing performed in the anterior AC processing stream. Subsequent studies are needed to investigate systematically how different nonspatial and spatial auditory tasks with varying task demands affect the distribution of activations in the human AC. Our results indicate that the dynamics of task-dependent modulations within human AC are considerably more complex than generally assumed. 


\section{References}

Alain C, Arnott SR, Hevenor S, Graham S, Grady CL (2001) "What" and "where" in the human auditory system. Proc Natl Acad Sci U S A 98:12301-12306

Arnott SR, Binns MA, Grady CL, Alain C (2004) Assessing the auditory dual-pathway model in humans. Neuroimage 22:401-408.

Bamiou DE, Musiek FE, Luxon LM (2003) The insula (Island of Reil) and its role in auditory processing. Literature review. Brain Res Brain Res Rev 42:143-154.

Barrett DJ, Hall DA (2006) Response preferences for "what" and "where" in human non-primary auditory cortex. Neuroimage 32:968-977.

Brechmann A, Gaschler-Markefski B, Sohr M, Yoneda K, Kaulisch T, Scheich H (2007) Working memory specific activity in auditory cortex: potential correlates of sequential processing and maintenance. Cereb Cortex 17:2544-2552.

Deary IJ, Simonotto E, Meyer M, Marshall A, Marshall I, Goddard N, Wardlaw JM (2004) The functional anatomy of inspection time: an event-related fMRI study. Neuroimage 22:1466-1479.

Degerman A, Rinne T, Salmi J, Salonen O, Alho K (2006) Selective attention to sound location or pitch studied with fMRI. Brain Res 1077:123-134.

Fritz JB, Elhilali M, Shamma SA (2005) Differential dynamic plasticity of A1 receptive fields during multiple spectral tasks. J Neurosci 25:7623-7635.

Gaab N, Gaser C, Schlaug G (2006) Improvement-related functional plasticity following pitch memory training. Neuroimage 31:255-263.

Griffiths TD, Warren JD (2002) The planum temporale as a computational hub. Trends Neurosci 25:348-353.

Hall DA, Plack CJ (2009) Pitch processing sites in the human auditory brain. Cereb Cortex 19:576-585.

Hall DA, Haggard MP, Akeroyd MA, Summerfield AQ, Palmer AR, Elliott MR, Bowtell RW (2000) Modulation and task effects in auditory processing measured using fMRI. Hum Brain Mapp 10:107-119.

Janata P, Tillmann B, Bharucha JJ (2002) Listening to polyphonic music recruits domain-general attention and working memory circuits. Cogn Affect Behav Neurosci 2:121-140.

Jäncke L, Mirzazade S, Shah NJ (1999) Attention modulates activity in the primary and the secondary auditory cortex: a functional magnetic resonance imaging study in human subjects. Neurosci Lett 266:125-128.

Kaas JH, Hackett TA (2000) Subdivisions of auditory cortex and processing streams in primates. Proc Natl Acad Sci U S A 97:11793-11799.

Krumbholz K, Eickhoff SB, Fink GR (2007) Feature- and object-based attentional modulation in the human auditory "where" pathway. J Cogn Neurosci 19:1721-1733.

Lawrence NS, Ross TJ, Hoffmann R, Garavan H, Stein EA (2003) Multiple neuronal networks mediate sustained attention. J Cogn Neurosci 15: $1028-1038$

Lomber SG, Malhotra S (2008) Double dissociation of 'what' and 'where' processing in auditory cortex. Nat Neurosci 11:609-616.

Martinkauppi S, Rämä P, Aronen HJ, Korvenoja A, Carlson S (2000) Working memory of auditory localization. Cereb Cortex 10:889-898.

Mayer AR, Franco AR, Canive J, Harrington DL (2009) The effects of stimulus modality and frequency of stimulus presentation on cross-modal distraction. Cereb Cortex 19:993-1007.
McKiernan KA, D’Angelo BR, Kaufman JN, Binder JR (2006) Interrupting the "stream of consciousness": an fMRI investigation. Neuroimage 29:1185-1191.

McLachlan N (2009) A computational model of human pitch strength and height judgments. Hear Res 249:23-35.

Merzenich MM, Knight PL, Roth GL (1975) Representation of cochlea within primary auditory cortex in the cat. J Neurophysiol 38:231-249.

Mutschler I, Wieckhorst B, Kowalevski S, Derix J, Wentlandt J, SchulzeBonhage A, Ball T (2009) Functional organization of the human anterior insular cortex. Neurosci Lett 457:66-70.

Patterson RD, Uppenkamp S, Johnsrude IS, Griffiths TD (2002) The processing of temporal pitch and melody information in auditory cortex. Neuron 36:767-776.

Penagos H, Melcher JR, Oxenham AJ (2004) A neural representation of pitch salience in nonprimary human auditory cortex revealed with functional magnetic resonance imaging. J Neurosci 24:6810-6815.

Petkov CI, Kang X, Alho K, Bertrand O, Yund EW, Woods DL (2004) Attentional modulation of human auditory cortex. Nat Neurosci 7 $658-663$.

Polley DB, Steinberg EE, Merzenich MM (2006) Perceptual learning directs auditory cortical map reorganization through top-down influences. J Neurosci 26:4970-4982.

Rauschecker JP, Tian B (2000) Mechanisms and streams for processing of "what" and "where" in auditory cortex. Proc Natl Acad Sci U S A 97:11800-11806.

Rinne T, Pekkola J, Degerman A, Autti T, Jääskeläinen IP, Sams M, Alho K (2005) Modulation of auditory cortex activation by sound presentation rate and attention. Hum Brain Mapp 26:94-99.

Rinne T, Balk MH, Koistinen S, Autti T, Alho K, Sams M (2008) Auditory selective attention modulates activation of human inferior colliculus. J Neurophysiol 100:3323-3327.

Romanski LM, Tian B, Fritz J, Mishkin M, Goldman-Rakic PS, Rauschecker JP (1999) Dual streams of auditory afferents target multiple domains in the primate prefrontal cortex. Nat Neurosci 2:1131-1136.

Smith SM, Jenkinson M, Woolrich MW, Beckmann CF, Behrens TEJ, Johansen-Berg H, Bannister PR, De Luca M, Drobnjak I, Flitney DE, Niazy RK, Saunders J, Vickers J, Zhang Y, De Stefano N, Brady JM Matthews PM (2004) Advances in functional and structural MR image analysis and implementation as FSL. Neuroimage 23 [Suppl 1]:S208-S219.

Warren JD, Griffiths TD (2003) Distinct mechanisms for processing spatial sequences and pitch sequences in the human auditory brain. J Neurosci 23:5799-5804

Wong PC, Parsons LM, Martinez M, Diehl RL (2004) The role of the insular cortex in pitch pattern perception: the effect of linguistic contexts. J Neurosci 24:9153-9160.

Woodruff PW, Benson RR, Bandettini PA, Kwong KK, Howard RJ, Talavage T, Belliveau J, Rosen BR (1996) Modulation of auditory and visual cortex by selective attention is modality-dependent. Neuroreport 7:19091913.

Woods DL, Stecker GC, Rinne T, Herron TJ, Cate AD, Yund EW, Liao I, Kang $\mathrm{X}$ (2009) Functional maps of human auditory cortex: effects of acoustic features and attention. PLoS One 4:e5183. 